\title{
Core-Selecting Auctions with Incomplete Information
}

\author{
Lawrence M. Ausubel and Oleg V. Baranov* \\ August 2010 \\ Preliminary
}

\begin{abstract}
Core-selecting auctions were proposed recently as alternatives to the VCG mechanism for environments with complementarities. While the Vickrey auction yields efficiency in pure private values models, this efficiency may come at the cost of extremely low seller revenues and a high vulnerability to collusion and shill bidding. By contrast, core-selecting auctions may result in "competitive" outcomes which seem to mitigate the problems of the Vickrey auction. Ausubel and Milgrom (2002), Ausubel, Cramton and Milgrom (2006), Day and Raghavan (2007), Day and Milgrom (2008) and Day and Cramton (2008) have advocated selling complementary goods using core-selecting auctions or two-stage procedures incorporating a core-selecting auction. Moreover, this research has been taken seriously by policymakers; for example, the UK has already implemented two spectrum auctions using the package clock auction, in which the second stage is a core-selecting auction (Cramton, 2009).
\end{abstract}

However, while the VCG mechanism is best motivated by its dominant-strategy property under incomplete information, the existing literature on core-selecting auctions performs only a completeinformation analysis. In this paper, we consider a simple incomplete-information model with two goods, two "local" bidders, and one "global bidder". We examine a parametric family of distributions in which, at one extreme, the local bidders' valuations are independent and, at the other extreme, the local bidders' valuations are perfectly correlated. We perform a full equilibrium analysis for four different coreselecting auction formats, including the nearest-Vickrey rule advocated by Day and Raghavan (2007) and Day and Cramton (2008), as well as the proxy auction of Ausubel and Milgrom (2002).

For the case of independent, uniformly-distributed valuations, we find that the VCG mechanism obtains $9 \%$ higher revenues and realizes 15\% greater efficiency than the best core-selecting auction that we analyze, similar to what was discovered simultaneously and independently by Goeree and Lien (2009). However, the comparison changes markedly when we introduce positive correlations in the local bidders' valuations. In particular, at the opposite extreme of perfect correlation, we find that the proxy auction obtains 33\% higher revenues and realizes the same efficiency as the VCG mechanism. Moreover, it seems appropriate to posit that substantial correlations in bidders' valuations would be present in important applications such as spectrum auctions. Thus, unlike Goeree and Lien, we conclude that there may be good reasons for policymakers to select a core-selecting auction rather than a VCG mechanism.

In the course of our analysis, we obtain closed-form solutions for the core-selecting pricing rules that we study. This enables us also to obtain revenue and efficiency rankings among the various rules. These rankings appear to be robust for the parameters of our model. The results may also provide useful guidance for policymakers.

\footnotetext{
* Department of Economics, University of Maryland, College Park, MD 20742, USA. Email: ausubel@econ.umd.edu and baranov@econ.umd.edu. We gratefully acknowledge the support of National Science Foundation Grant SES-09-24773. We are also grateful to Paul Milgrom, Peter Cramton, John Rust and Daniel Vincent for valuable conversations and to seminar participants at Maryland, Carnegie Mellon and the Econometric Society World Congress for helpful comments. All errors are our own.
} 


\section{Introduction}

Core-selecting auctions have recently been proposed as alternatives to the Vickrey-Clarke-Groves (VCG) mechanism. In the VCG mechanism, the items are allocated so as to maximize revenues subject to the feasibility of the selected bids and each bidder is charged the opportunity cost of receiving the allocated items. While the mechanism has the attractive property that truth-telling is a dominant strategy — and truth-telling by all participants in the VCG mechanism implies efficient outcomes - there are several reasons to be wary of VCG in environments with complementarities. First, the VCG mechanism may generate low revenues (and, in environments with extreme complementarities, the revenues may equal zero). Second, VCG outcomes may be non-monotonic in the sense that increasing the number of bidders or increasing their valuations may reduce the seller's revenues. Third, the VCG mechanism may be especially vulnerable to unusual forms of collusive behavior, including collusion by losing bidders and shill bidding.

The simplest environment in which these issues can arise has just two items, "East" and "West" (which may be thought of as spectrum licenses for the Eastern half and Western half of a country), and three bidders. The "global" bidder views East and West as perfect complements, valuing the package \{East, West $\}$ at 1, but obtaining no value from either item individually. Meanwhile, local bidder 1 values East at 1, but obtains no value from West; and local bidder 2 values West at 1, but obtains no value from East. Observe that the VCG mechanism ${ }^{1}$ allocates East to local bidder 1 and West to local bidder 2, maximizing social surplus at 2. However, the mechanism charges a price of zero to each bidder. ${ }^{2}$ The VCG outcome is non-monotonic in that, if each of the local bidders' values declined from 1 to $1 / 2$, the seller's revenues would increase from 0 to 1 . The explanation for this non-monotonicity, as well as for the opportunities present for loser collusion and shill bidding, is that the VCG outcome may lie outside the core; ${ }^{3}$ with the data of this paragraph, a coalition of the seller and the global bidder can block the allocation at zero prices to the local bidders. ${ }^{4}$

Observe that the potential deficiencies of the VCG mechanism are likely to be empirically relevant. In the first place, much of the motivation for allowing package bidding in auctions arises from environments where there appear to be strong complementarities among items. Furthermore, in the area of telecommunications spectrum auctions, empirical work suggests that there exist substantial synergies among licenses covering different geographic areas. ${ }^{5}$ Similarly, there is a growing interest in auctions with package bidding for financial assets, and this again occurs in environments where there are apparent complementarities among assets.

\footnotetext{
${ }^{1}$ The VCG mechanism was developed in the work of Vickrey (1961), Clarke (1971) and Groves (1973). Throughout this paper, we will use the terms "VCG mechanism" and "Vickrey auction" interchangeably.

${ }^{2}$ Observe that the total surplus when local bidder 1 is absent equals 1 , and so the incremental surplus created by local bidder 1 equals 1 . Similarly, local bidder 2's incremental surplus also equals 1. In the VCG mechanism, each bidder is permitted to retain the entire incremental surplus that she creates, implying that the price paid by each local bidder is zero.

${ }^{3}$ The core is the subset of allocations in payoff space that are feasible and unblocked by any coalition.

${ }^{4}$ See Ausubel and Milgrom (2002).

${ }^{5}$ See, for example, Ausubel, Cramton, McAfee and McMillan (1997) and Fox and Bajari (2009).
} 
As a result of this critique, researchers and auction practitioners recently began to explore a class of alternative mechanisms that have become known as core-selecting auctions. As in the VCG mechanism, buyers submit bids associated with various subsets of the set of all items, and the auctioneer determines the combination of bids which maximizes total revenues subject to feasibility. However, as seen two paragraphs above, applying the VCG payment rule in a complements environment may yield a profit allocation that lies outside the core. Instead, a core-selecting auction uses a different pricing rule — a rule always requiring the same or higher payments — which assures that the outcome is always in the core relative to the reported values.

Despite the very recent development of core-selecting auctions, they have already been selected for some important applications. Ofcom, the UK telecommunications regulator, has conducted two spectrum auctions using a "package clock” auction: a two-stage auction procedure in which a simultaneous ascending clock phase is followed by a sealed-bid package auction. For the second stage, Ofcom utilized a core-selecting auction with the nearest-Vickrey pricing rule. ${ }^{6}$ The US Federal Aviation Administration (FAA) recently planned slot auctions for landing rights at the three New York City airports. While these auctions were stopped by an airline-industry lawsuit, the published regulations included the use of a core-selecting auction with the nearest-Vickrey pricing rule. ${ }^{7}$

However, to date, most studies of package bidding have been limited to complete-information analyses. This is not a particularly satisfying state of affairs, as much of the motivation for using VCG or other package-bidding mechanisms is that bidders possess incomplete and asymmetric information. At the same time, it is easy to understand why the shortcut of assuming complete information has typically been taken: analyses of auctions under incomplete information can be extremely intricate, except when truth-telling is an equilibrium. Moreover, the typical sort of environment motivating package bidding inherently includes asymmetries, as some bidders desire smaller sets of items and other bidders desire larger sets of items. Researchers have found that asymmetric auctions are particularly difficult to analyze.

A few contemporaneous papers have introduced explicit incomplete-information analyses of package bidding, but they are limited to considering independent valuations. Independence is an extremely confining assumption in an auction environment. In many of the most important applications of package bidding, such as spectrum auctions, we would expect there to be significant correlations among bidders' signals - and correlation among bidders' signals has been one of the important ingredients in the theory of auctions of single items. Moreover, a central message of auction theory and mechanism design is that, when correlations are present, particular choices of auction format may enhance the ability of the seller to extract revenues from bidders. ${ }^{8}$

The current paper seeks to advance the analysis of package bidding. We consider a very simple and stylized class of models in which one bidder values the items as perfect complements. We compare

\footnotetext{
${ }^{6}$ See Cramton (2009).

${ }^{7}$ See Federal Aviation Administration (2008).

${ }^{8}$ See, for example, Milgrom and Weber (1982) and Crémer and McLean (1985).
} 
and contrast a variety of package bidding formats, including the core-selecting auctions in the literature, as well as the VCG mechanism.

Our model is an incomplete-information version of the auction environment with two items and three bidders that is described in the second paragraph of this Introduction. The global bidder obtains value $u$ from winning both the Eastern and Western licenses, but gets zero value from having only East or West. Local bidder 1 values East at $v_{1}$, but obtains no value from West; while local bidder 2 values West at $v_{2}$, but obtains no value from East. The game is a standard Bayesian game in which each player knows the realization of her own value, but only the distribution from which her opponents' values were drawn. The players simultaneously and independently submit bids, where $b_{1}$ denotes the bid submitted by local bidder 1 for East, $b_{2}$ denotes the bid submitted by local bidder 2 for West, and $B$ denotes the package bid submitted by the global bidder for \{East, West . The solution concept is Bayesian-Nash equilibrium. $^{9}$

One of the novel aspects of our analysis is the family of distributions that we treat. The local bidders' values are perfectly correlated with probability $\gamma$ and independently distributed with probability $1-\gamma$. (Moreover, at the time that the local bidder selects her bid, she is unaware of whether the values are perfectly correlated or independent.) Thus, we consider a parameterized family of distributions that permits the correlation between local bidders' signals to be varied continuously from zero to one. Surprisingly, despite the private information and correlated signals, we are able to obtain explicit closed-form solutions for the core-selecting auction formats considered - for all $\gamma \in[0,1]$. And the possibility of positive correlation has a quite substantial impact on our comparison of the various package-bidding formats.

To see the various package-bidding mechanisms that we compare and contrast, suppose that the bids submitted by the respective bidders are $b_{1}=6, b_{2}=8$, and $B=10$. In any of the mechanisms, the auctioneer first solves the winner determination problem of finding the allocation which maximizes revenues subject to the feasibility constraint. This bid data clearly results in local bidder 1 winning East and local bidder 2 winning West, as $6+8=14>10$. The payments, $p_{1}$ and $p_{2}$, of local bidders 1 and 2, respectively, remain to be defined. The various mechanisms to be discussed in this paper will differ in their payment rules. Our analysis will consider the following package-bidding mechanisms:

VICKREY-ClARKE-GROVES (VCG): Payments are determined such that each winner receives a payoff equal to the incremental surplus that she brings to the system. The incremental surplus of local bidder 1 equals 4, as surplus (evaluated using the bidders' bids) equals 14 if local bidder 1 is present, and 10 (the global bidder's value) if local bidder 1 is absent. Thus, $b_{1}-p_{1}=4 \Rightarrow p_{1}=2$. Similarly, the incremental surplus of local bidder 2 equals 4 , so $b_{2}-p_{2}=4 \Rightarrow p_{2}=4$. Thus, the VCG payments are $\left(p_{1}, p_{2}\right)=(2,4)$. However, this outcome is not in the core, as the seller and the global bidder form a blocking coalition: together, they

\footnotetext{
${ }^{9}$ Further, the joint distribution of values will be symmetric with respect to the two local bidders, and we will limit attention to Bayesian-Nash equilibria that are symmetric with respect to the two local bidders. In addition, in all of the core-selecting auctions, the global bidder will have a weakly-dominant strategy, and we will then limit attention to Bayesian-Nash equilibria in which the global bidder plays her weakly-dominant strategy.
} 
can realize surplus of 10 (the global bidder's value), while in the VCG outcome, the seller receives payoff of $6=p_{1}+p_{2}$ and the global bidder receives payoff of 0 .

NEAREST-VICKREY: Payments are determined such that the profit allocation is the bidderoptimal core allocation that minimizes the Euclidean distance from the VCG outcome. In order to avoid the presence of any blocking coalitions, the payments, $p_{1}$ and $p_{2}$, must sum to at least 10; and in a bidder-optimal core allocation, the payments must sum to exactly 10 . The payments that minimize the distance from the VCG payments of $(2,4)$ are $\left(p_{1}, p_{2}\right)=(4,6)$.

PROXY Auction: Payments are determined that reflect the outcome of "proxy agents" competing in a simultaneous ascending auction with package bidding and arbitrarily small bid increments, $\varepsilon$. The bids $b_{1}, b_{2}$ and $B$ are reinterpreted as limit prices that the bidders have given their respective proxy agents. Each proxy agent must bid in the "virtual auction" whenever it is not a provisionally-winning bidder. In the initial round, all three proxy agents submit bids of $\varepsilon$, making the two local bidders provisional winners. In round two, the proxy agent for the global bidder raises its bid to $3 \varepsilon$, making the global bidder the provisional winner; in round three, the proxy agents for each of the local bidders raise their bids to $2 \varepsilon$, making the two local bidders provisional winners; and the process repeats until the proxy agent for the global bidder drops out of the auction at a price of essentially $B=10$. Thus, the local bidders win the virtual auction at prices of essentially $\left(p_{1}, p_{2}\right)=(5,5)$, an alternative bidder-optimal core outcome.

Proportional Pricing: Payments are determined such that the bids are scaled down, proportionally, until the bidder-optimal frontier of the core is reached. In the above example, the bids of the local bidders sum to 14 , and so they can each be scaled down by a factor of 5/7 in order to sum to 10 . Thus, the payments are $\left(p_{1}, p_{2}\right)=(30 / 7,40 / 7)$.

NEAREST-BID: Payments are determined such that the profit allocation is the bidder-optimal core allocation that minimizes the Euclidean distance from the vector of winning bids. In the above example, the bidder-optimal core payments that minimize the distance from the winning bids of $(6,8)$ are $\left(p_{1}, p_{2}\right)=(4,6)$, coinciding with the nearest-Vickrey outcome in this example.

FIRST-PRICE PACKAGE: Payments simply correspond to the amounts of the winning bids. That is, the auctioneer first solves the winner determination problem of finding the allocation which maximizes revenues subject to the feasibility constraint; and the winning bidders' required payments are simply the amounts of their winning bids. If the same bids were submitted as in the above example, then the payments would be $\left(p_{1}, p_{2}\right)=\left(b_{1}, b_{2}\right)=(6,8)$. Obviously, since this is a "first price" rather than a "second price" auction format, it should be expected that bids would be substantially different from those in the other core-selecting auctions. 
Each of these package-bidding pricing rules, as applied to the bid data $b_{1}=6, b_{2}=8$, and $B=10$, is illustrated in Figure 1. Observe that the set of prices associated with core allocations is the shaded triangle of this figure, while the set of bidder-optimal core prices is the hypotenuse of this triangle.

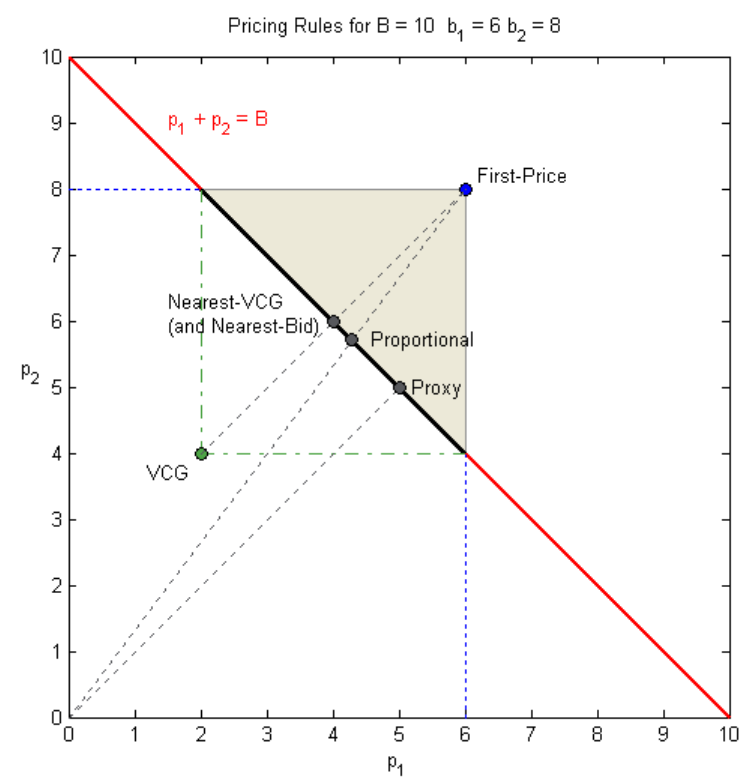

Figure 1. Package-Bidding Pricing Rules (as applied to example bid data)

For the various "second-price-like" core-selecting auctions, if the marginal distribution of each bidder's value is the uniform distribution, then we are able to derive explicit closed-form solutions for equilibria, for all $\gamma \in[0,1]$. However, for the first-price package auction, the methodology of this paper does not yield a solution. To compare the performance of the first-price package auction with the other core-selecting auctions, we report the revenues and efficiency as computed using a numerical technique for approximating equilibria that is introduced in Baranov (2010).

The VCG mechanism was introduced in the classic theory of auctions and public choice. William Vickrey (1961) treated auctions with multiple units of a homogeneous product, while Edward Clarke (1971) and Theodore Groves (1973) treated public choice problems. The Clarke-Groves treatment subsumed the environment that Vickrey studied as well as auctions of multiple heterogeneous objects. We use the terminology "VCG mechanism” and "Vickrey auction” interchangeably.

Our treatment of the proxy auction is based on Ausubel and Milgrom (2002, 2006). There the "ascending proxy auction" design is described; it is proven that this auction generally yields a core allocation with respect to the preferences reported by the bidders and (subject to a refinement) the set of complete-information equilibrium allocations coincides with the set of bidder-optimal core allocations. A closely-related auction procedure was developed independently by Parkes and Ungar (2000) and Parkes (2001). A two-stage auction procedure comprising a (multi-round) ascendingclock auction followed by a single proxy auction round was proposed by Ausubel, Cramton and 
Milgrom (2006). This became the basis for the "package clock” design recently adopted by the UK government for spectrum auctions (Cramton, 2009).

Hoffman, Menon, van den Heever and Wilson (2006) introduced acceleration techniques for computing the proxy auction. Day and Raghavan (2007) propose a modification to the proxy auction where the "virtual" auctions of the proxy are superseded by a direct computation of bidder-optimal core allocation — in particular, they advocate the bidder-optimal core allocation that minimizes the maximum deviation from the VCG payments. They also introduce a core constraint generation algorithm which is an especially effective method for calculating bidder-optimal core allocations. Day and Milgrom (2008) define the class of core-selecting auctions and prove that, among coreselecting auctions, the ones that minimize seller revenues also maximize incentives for truthful reporting and, in contrast to the Vickrey auction, yield monotonicity of revenues in the number of bidders and their bids. Day and Cramton (2009) propose the nearest-Vickrey pricing rule and demonstrate how to compute it efficiently.

Three other recent papers have begun to explore the comparison among core-selecting auctions. Erdil and Klemperer (2009) define a class of payment rules referred to as "reference rules" — the proxy auction's payment rule is one example, while the nearest-Vickrey rule is not - and they argue that reference rules reduce the marginal incentive to deviate as compared to other payment rules. While their paper does not explicitly contain incomplete-information analysis, their conclusions foreshadow the results of the current paper. Goeree and Lien (2009) consider the incomplete-information game with a global bidder and two local bidders whose valuations are independent and uniformlydistributed. Simultaneously and independently from the current paper, they solve for the BayesianNash equilibrium of the nearest-Vickrey pricing rule for independent uniform distributions and they find that the VCG mechanism dominates it in expected revenues as well as efficiency. Sano (2010) considers the incomplete-information game with a global bidder and two local bidders whose valuations are independent and uniformly-distributed. Simultaneously and independently from the current paper, he solves for the Bayesian-Nash equilibrium of the proxy auction under independence, finding that high-value local bidders submit almost their true values, while low-value local bidders shade considerably.

In the current paper, we too analyze incomplete-information games with a global bidder and two local bidders. We formulate the game and solve for equilibria, allowing independence $(\gamma=0)$ or correlation $(\gamma>0)$ between the local bidders' values. We consider four different core-selecting auctions - the nearest-Vickrey, the proxy, the proportional and the nearest-bid pricing rule — and for each $\gamma \in[0,1]$, we are able to obtain explicit closed-form solutions under certain assumptions on the distributions. ${ }^{10}$ For the case where the marginal distributions are uniform, we obtain Figure 2, which summarizes the expected seller revenues and efficiency in the equilibrium:

\footnotetext{
${ }^{10}$ Only three solutions are required, as the equilibrium for the proportional pricing rule coincides with the equilibrium for the nearest-Vickrey rule in the model we consider.
} 

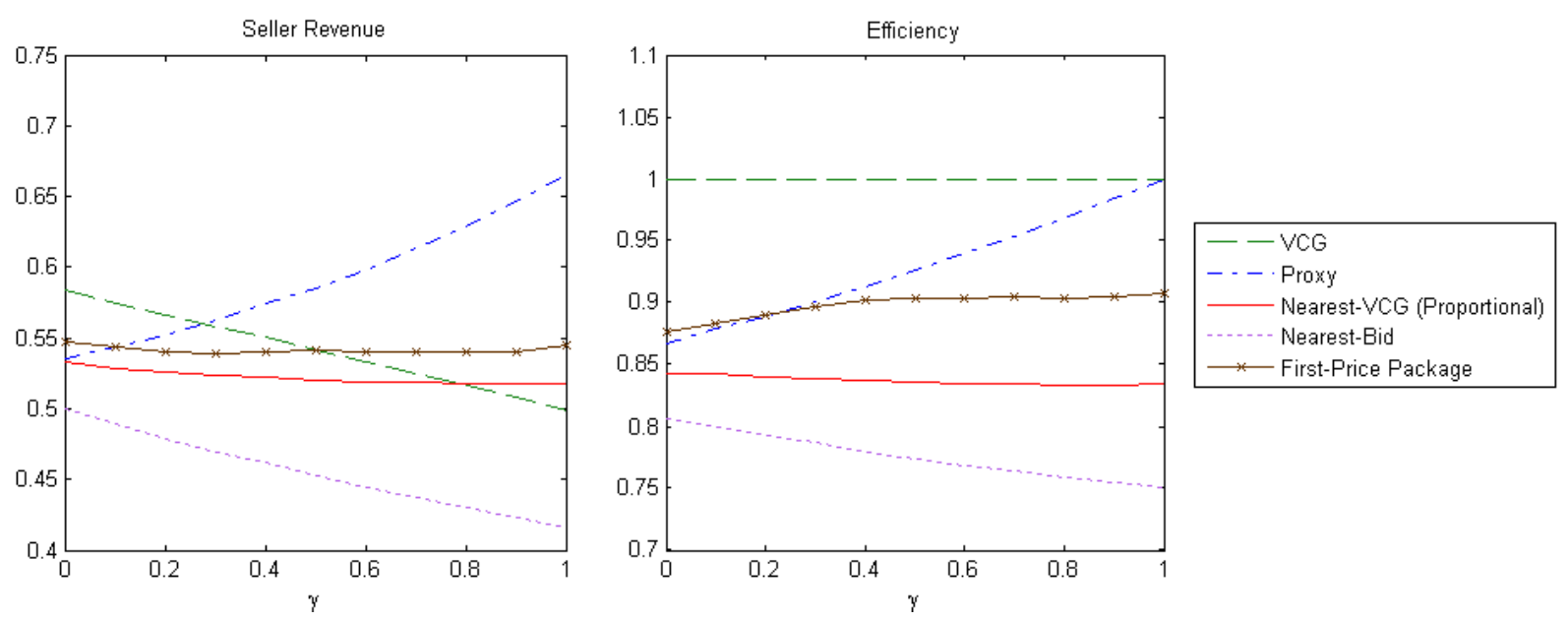

Figure 2. Seller Revenue and Efficiency for $\alpha=1$ and all $\gamma \in[0,1]$

Counter to Goeree and Lien, we find that the choice between a core-selecting auction or the VCG mechanism is sensitive to the information structure. As shown in Figure 2, the relative performance of the alternative mechanisms changes substantially as the correlation $\gamma$ increases from zero to 1 . When $\gamma=0$, the VCG mechanism raises $8.9 \%$ higher expected revenues than the proxy auction, and it achieves $9.6 \%$ higher revenue than the nearest-Vickrey rule, despite achieving greater efficiency. However, at the opposite extreme, when $\gamma=1$, the proxy auction attains $33.3 \%$ higher expected revenues than the VCG mechanism while also realizing full efficiency. Clearly, the choice of whether to use the VCG mechanism or a core-selecting auction depends on the likely informational environment.

We also consider the effect of varying the uniform distribution on the local bidders' values. While the distribution $F(v)=v^{\alpha}$ does not generally admit a closed-form solution, it does when $\alpha=2$. Stable numerical simulations can be found for other $\alpha$ - for symmetry with $\alpha=2$, we also consider $\alpha=1 / 2$. In this formulation, $\alpha$ controls the relative frequency of local bidders' winnings under full efficiency. For example, when $\alpha=1$ (uniform distribution) local bidders are expected to win with probability $1 / 2$ while they only expected to win with probability $1 / 3$ when $\alpha=1 / 2$. We find that the comparison among the VCG mechanism and the various core-selecting auctions changes in $\alpha$. In particular, the case of $\alpha=2$ reverses the revenue ranking of the proxy auction and the VCG mechanism, while the case of $\alpha=1 / 2$ enhances the revenue advantage of VCG emphasized by Goeree and Lien.

This paper proceeds as follows. In Section 2, we present the model, including the family of distributions that allow partial correlation, and we detail the package-bidding mechanisms to be considered. In Section 3, we introduce the pivotal pricing property and we establish Lemma 3, which provides local optimality conditions for local bidders in any mechanism satisfying the pivotal pricing property. We solve for explicit closed-form solutions for the various mechanisms under consideration, for all correlation parameters, in Section 4. In Section 5, we discuss extensions to the basic model, and we conclude in Section 6. Most proofs are relegated to Appendix A, and the solutions for different values of parameters $\gamma$ and $\alpha$ are summarized in Appendix B. 


\section{The Model}

Two items are offered for sale. There are two local bidders, 1 and 2, who are interested in only one item and receive no extra utility from acquiring the second item. Their values are denoted $v_{1}$ and $v_{2}$, respectively. There is one global bidder who wants to acquire both items and obtains no utility from owning just one item. Her value for the pair of items is denoted $u$. The bidders are risk neutral and have quasilinear utilities: the payoff of local bidder $i$, if she wins one unit at price $p_{i}$, is $v_{i}-p_{i}$; and the payoff of the global bidder, if she wins both units at a total price of $p$, is $u-p$.

The value, $u$, of the global bidder is independently drawn from the distribution on $[0,2]$ described by a cumulative distribution function $G(u)$ with atomless probability density function $g(u)$. With probability $\gamma$, the values, $v_{i}(i=1,2)$, of local bidders are perfectly correlated and drawn from a distribution on the interval $[0,1]$, defined by a cumulative distribution function $F(v)$ with atomless density $f(v)$. With probability $1-\gamma$, the values of the local bidders are independently drawn from the same distribution $F(v)$.

The assumption of independence between value realizations of the global bidder versus the local bidders seems reasonable enough - the scale of operations, cost structure and other bidder-specific characteristics of the global and local bidders may be substantially unrelated. However, it seems likely that there might be positive correlation between one local bidder's value and another. For example, in a spectrum auction, the local bidders might be two firms that intend to deploy identical telecommunications technologies in different geographic regions.

Parameter $\gamma$ controls the amount of correlation between the local bidders' values. The local bidders' value model is summarized by the conditional cumulative distribution function of the local bidder $i$ given her value $v_{i}$ :

$$
F_{L}\left(v_{j} \mid v_{i}=x\right)=\left\{\begin{array}{cc}
(1-\gamma) F\left(v_{j}\right), & \text { if } v_{j}<x \\
(1-\gamma) F\left(v_{j}\right)+\gamma, & \text { if } v_{j} \geq x
\end{array} \text { for } i \neq j .\right.
$$

It is interesting to observe that $v_{i}$ and $v_{j}$ are not affiliated random variables for any $\gamma>0 .{ }^{11}$ Nevertheless, for $y \geq x, F_{L}(\bullet \mid y)$ (first-order) stochastically dominates $F_{L}(\bullet \mid x)$. The failure of affiliation would prevent some of the results in the theory of single-item auctions from going through, but note that the structure of winning is different in the current package-bidding context: local bidder 1's bid need not exceed the bid of local bidder 2; rather the sum of the bids of local bidders 1 and 2 needs to exceed the bid of the global bidder.

Our model handles both homogeneous and heterogeneous environments. In the former interpretation, local bidder $i$ derives positive utility $v_{i}$ from winning either item. ${ }^{12}$ In the latter interpretation, there

\footnotetext{
${ }^{11}$ Consider $x>y>z$ and let $\Xi(\cdot, \cdot)$ denote the joint probability of $v_{i}$ and $v_{j}$. Then $(y, y) \vee(x, z)=(x, y)$ and $(y, y) \wedge(x, z)=$ $(y, z)$, but $\Xi(x, y) \Xi(y, z)<\Xi(y, y) \Xi(x, z)$, contradicting the affiliation inequality.

${ }^{12}$ Then the global bidder exhibits classic increasing returns to scale.
} 
are two heterogeneous items, East and West; local bidder 1 obtains positive utility only from East and local bidder 2 obtains positive utility only from West. ${ }^{13}$ Our equilibrium solutions are fully consistent with either interpretation.

All of the auction mechanisms that we analyze in this paper, other than the VCG mechanism, satisfy the following definition:

Definition 1. A core-selecting auction is a mapping from bids to allocations and payments such that the payoffs resulting from every bid profile are elements of the core. ${ }^{14}$

Without loss of generality we limit our attention to the restricted auctions in which each bidder is allowed to submit only one bid. While impractical in a general environment, this limitation does not affect efficiency in any way because of the perfect complementarity nature of the bidders' preferences in the model we consider here. For example, the global bidder has value for a package of two items and her bid $B$ is interpreted as a package bid for two items. Each local bidder $i$ is interested only in one item and her bid $b_{i}$ expresses her willingness to pay up to $b_{i}$ for the one item.

All auctions considered in the paper proceed in the following manner. First, all bidders submit their bids to the auctioneer who then chooses an allocation which maximizes total welfare with respect to the bids. In our simple model, only two outcomes are possible. If the package bid of the global bidder is greater than the sum of the local bids, i.e. $B>b_{1}+b_{2}$, the global bidder wins the auction and receives both items. The local bidders win the auction and each receive one item whenever the sum of their bids is higher than the package bid of the global bidder, i.e. $B<b_{1}+b_{2}$. Ties are resolved using a fair randomizing device. The payment each winner is required to make depends on a specific pricing rule.

We consider the VCG mechanism and several core-selecting pricing rules. Denote $V_{1}$ and $V_{2}$, the VCG payments of local bidders in case of winning, i.e. $V_{1}=\max \left\{0, B-b_{2}\right\}, \quad V_{2}=\max \left\{0, B-b_{1}\right\}$. Additionally, we use $p\left(b_{1}, b_{2}, B\right)$ to denote a payment vector associated with the corresponding bids $b_{1}, b_{2}$ by local bidders and a bid $B$ by the global bidder.

Without loss of generality, we will assume that $b_{1} \geq b_{2}$.

(1) VCG Mechanism (Benchmark Rule)

This is a well-known pricing rule which is motivated by its dominant strategy property. Under this rule, the payment of the particular bidder does not depend upon her bid and only affects the allocation.

$$
p\left(b_{1}, b_{2}, B\right)=\left\{\begin{array}{cc}
\left(V_{1}, V_{2}, 0\right) & \text { if } \mathrm{B}<b_{1}+b_{2} \\
\left(0,0, b_{1}+b_{2}\right) & \text { if } \mathrm{B}>b_{1}+b_{2}
\end{array}\right.
$$

\footnotetext{
${ }^{13}$ Then the global bidder is intending to implement a technology which (for technical or marketing reasons) is only economical if deployed on a nationwide basis.

${ }^{14}$ This definition is taken from Day and Milgrom (2008).
} 
(2) Proxy Auction

The ascending proxy auction was suggested by Ausubel and Migrom (2002). Given our simple model, it can be summarized using the following formula:

$$
p\left(b_{1}, b_{2}, B\right)=\left\{\begin{array}{ccc}
\left(\frac{1}{2} B, \frac{1}{2} B, 0\right) & \text { if } & 2 b_{2}>B \\
\left(B-b_{2}, b_{2}, 0\right) & \text { if } & 2 b_{2} \leq B<b_{1}+b_{2} \\
\left(0,0, b_{1}+b_{2}\right) & \text { if } & B>b_{1}+b_{2}
\end{array}\right.
$$

(3) Nearest-VCG Rule

The nearest-VCG pricing rule was introduced by Day and Raghavan (2007) and Day and Milgrom (2008). The central idea of this rule is to select the bidder-optimal core allocation that minimizes the distance to the VCG point.

$$
\begin{aligned}
& p\left(b_{1}, b_{2}, B\right)=\left\{\begin{array}{ccc}
\left(V_{1}+\Delta, V_{2}+\Delta, 0\right) & \text { if } & \mathrm{B}<b_{1}+b_{2} \\
\left(0,0, b_{1}+b_{2}\right) & \text { if } & \mathrm{B}>b_{1}+b_{2}
\end{array}\right. \\
& \text { where } \Delta=\frac{B-V_{1}-V_{2}}{2}
\end{aligned}
$$

(4) Proportional Rule

This is a natural rule to consider in this environment. Whenever the local side wins the auction, they split the amount they are required to pay proportionally to their bids.

$$
p\left(b_{1}, b_{2}, B\right)=\left\{\begin{array}{cc}
\left(\frac{b_{1}}{b_{1}+b_{2}} B, \frac{b_{2}}{b_{1}+b_{2}} B, 0\right) & \text { if } B<b_{1}+b_{2} \\
\left(0,0, b_{1}+b_{2}\right) & \text { if } B>b_{1}+b_{2} .
\end{array}\right.
$$

(5) Nearest-Bid Rule

The "nearest-bid" description corresponds to the point in a minimum-revenue core which is the closest to the winners' bids. This rule can be motivated by a simple description of the payment procedure. In case of winning each local bidder pays her bid and then gets a refund. The amount of the refund is just half of the "money left on the table", i.e. $b_{1}+b_{2}-B$. As with Proxy Rule, if bids are too different, the amount of refund might be higher than the smallest of the locals' bids. Since payments can not be negative, the local bidder $i$ with the small bid $\left(b_{i} \ll b_{j}\right)$ is reimbursed completely while the local bidder $j$ pays the global bidder's bid alone. This rule is intuitive and easy to explain to the bidders.

$$
\begin{aligned}
& p\left(b_{1}, b_{2}, B\right)=\left\{\begin{array}{ccc}
\left(b_{1}-\Delta, b_{2}-\Delta, 0\right) & \text { if } & b_{1}-b_{2}<B<b_{1}+b_{2} \\
(B, 0,0) & \text { if } & b_{1} \geq B+b_{2} \\
\left(0,0, b_{1}+b_{2}\right) & \text { if } & B>b_{1}+b_{2},
\end{array}\right. \\
& \text { where } \Delta=\frac{b_{1}+b_{2}-B}{2} .
\end{aligned}
$$




\section{Initial Analysis}

Definition 2. A bid $b$ by bidder $i$ is pivotal if, for any $\varepsilon>0$, a bid $b+\varepsilon$ yields bidder $i$ a non-empty set of items, while a bid of $b-\varepsilon$ yields bidder $i$ the empty set.

Note that, in auctions with pricing rules (1)-(5), any bid $\left(b_{1}, b_{2}\right.$ or $\left.B\right)$ is pivotal if and only if $b_{1}+b_{2}=B$.

Definition 3. An auction satisfies the pivotal pricing property with respect to a given bidder if, whenever the bidder's bid is pivotal, the price that she pays (if she wins) equals her bid.

The pivotal pricing property is very natural and is satisfied for the most of the reasonable auction formats. Consider standard single-item auction with at least three bidders. First-price and all-pay auctions necessarily satisfy the pivotal pricing property since the winner always pays her bid. In a second-price auction a winning bid is pivotal only if top two bids are equal to each other in which case the winner pays her bid precisely. However, some auctions do not satisfy this property. For example, in a third-price auction a winner with pivotal bid in general pays less then her bid.

Lemma 0. The VCG mechanism satisfies the pivotal pricing property with respect to all bidders.

Proof: If bidder $i$ 's bid, $b_{i}$, is pivotal, then the incremental surplus contributed by bidder $i$ is zero. By the specification of the VCG mechanism, bidder i's payoff in the mechanism equals zero. Consequently, bidder $i$ pays a price of $b_{i}$.

Lemma 1. Every core-selecting auction satisfies the pivotal pricing property with respect to all bidders.

Proof: Let $p_{i}$ denote the price paid by bidder $i$ when her bid, $b_{i}$, is pivotal, and let $S_{i}$ denote the set of winning bidders if bidder $i$ had instead submitted a bid of $b_{i}-\varepsilon$. By the definition of a pivotal bid, $i \notin S_{i}$. Suppose that $p_{i}<b_{i}$. Then the allocation can be blocked by the coalition comprising the seller and set $S_{i}$. Suppose instead that $p_{i}>b_{i}$. Then the allocation can be blocked by the coalition comprising bidder $i$ alone. We conclude that $p_{i}=b_{i}$.

Lemma 2. The global bidder has a weakly dominant strategy to bid her value in auctions with pricing rules $(1)-(5)$.

Proof: For each of these pricing rules, the global bidder wins if and only if her package bid, $B$, satisfies $B \geq b_{1}+b_{2}$, and her payment is then $b_{1}+b_{2}$. Consequently, the exact same argument holds as in the standard second-price auction for a single item. 
In what follows, we assume that the global bidder bids according to her weakly dominant strategy, i.e. $B(u)=u$.

With a slight abuse of notation let $\beta$ (.) denote the symmetric equilibrium bid function of the local bidders for all pricing rules. Additionally, denote $\Phi_{i}\left(b_{i}, v_{i}\right)$ and $\varphi_{i}\left(b_{i}, v_{i}\right)$ the probability of winning and marginal probability of winning for a local bidder $i$ who submits a bid $b_{i}$ assuming all other bidders follow their equilibrium strategies, i.e.:

$$
\begin{aligned}
\Phi_{i}\left(b_{i}, v_{i}\right) & =\operatorname{Pr}\left(b_{i}+b_{j}>B\right)= \\
& =\gamma \int_{b_{i}+\beta\left(v_{i}\right)>B(u)} g(u) d u+(1-\gamma) \iint_{b_{i}+\beta\left(v_{j}\right)>B(u)} f\left(v_{j}\right) g(u) d v_{j} d u= \\
& =\gamma G\left(b_{i}+\beta\left(v_{i}\right)\right)+(1-\gamma) \int_{v_{j}} f\left(v_{j}\right) G\left(b_{i}+\beta\left(v_{j}\right)\right) d v_{j} \\
\varphi_{i}\left(b_{i}, v_{i}\right) & =\frac{\partial \Phi_{i}\left(b_{i}, v_{i}\right)}{\partial b_{i}} \\
& =\gamma g\left(b_{i}+\beta\left(v_{i}\right)\right)+(1-\gamma) \int_{v_{j}} f\left(v_{j}\right) g\left(b_{i}+\beta\left(v_{j}\right)\right) d v_{j} .
\end{aligned}
$$

Let $P_{i}\left(b_{i}, v_{i}\right)$ and $M P_{i}\left(b_{i}, v_{i}\right)$ denote the expected payment and the expected marginal payment, respectively, for a local bidder who submits a bid $b_{i}$ assuming all other bidders follow their equilibrium strategies, i.e.:

$$
\begin{aligned}
P_{i}\left(b_{i}, v_{i}\right)= & E p_{i}\left(b_{i}, b_{j}, B\right)= \\
= & \gamma \int_{u} p_{i}\left(b_{i}, \beta\left(v_{i}\right), u\right) g(u) d u+(1-\gamma) \iint_{u, v_{j}} p_{i}\left(b_{i}, \beta\left(v_{j}\right), u\right) f\left(v_{j}\right) g(u) d v_{j} d u \\
M P_{i}\left(b_{i}, v_{i}\right) & =E p_{i}^{\prime}\left(b_{i}, b_{j}, B\right)= \\
& =\gamma \int_{u} p_{i}^{\prime}\left(b_{i}, \beta\left(v_{i}\right), u\right) g(u) d u+(1-\gamma) \iint_{u, v_{j}} p_{i}^{\prime}\left(b_{i}, \beta\left(v_{j}\right), u\right) f\left(v_{j}\right) g(u) d v_{j} d u .
\end{aligned}
$$

Lemma 3. For an auction satisfying the pivotal pricing property, the optimality conditions for a local bidder $i$ are given by:

$$
\begin{aligned}
& \left(v_{i}-b_{i}\right) \varphi_{i}\left(b_{i}, v_{i}\right) \leq M P_{i}\left(b_{i}, v_{i}\right) \quad b_{i} \geq 0 \\
& b_{i}\left[\left(v_{i}-b_{i}\right) \varphi_{i}\left(b_{i}, v_{i}\right)-M P_{i}\left(b_{i}, v_{i}\right)\right]=0 .
\end{aligned}
$$

Proof: See Appendix A.

Lemma 3 just simplifies the Karush-Kuhn-Tucker conditions for the local bidders’ profit maximization problem taking into account the pivotal pricing property. Intuitively, an infinitely small 
increase in a bid affects costs by increasing expected payment in non-pivotal states and adding a new payment in the pivotal state (when the increase results in a pivotal bid, or the state in which bidder wins only because she increased her bid by a small amount ). The latter payment equals to the player's bid according to the pivotal pricing property.

In case $\varphi_{i}\left(b_{i}, v_{i}\right)>0$, the optimality conditions in Lemma 3 can be rewritten as:

$$
b_{i}=\max \left(0, v_{i}-\frac{M P_{i}\left(b_{i}, v_{i}\right)}{\varphi_{i}\left(b_{i}, v_{i}\right)}\right) \text {. }
$$

Note that a local bidder shades her bid when the expected marginal payment is positive. We formalize this general functional form of the equilibrium local's bidder bid function in a Corollary 1.

Corollary 1. The general functional form of the locals' equilibrium bid function is

$$
\begin{aligned}
& \beta(v)=\left\{\begin{array}{cc}
0 & v \leq d(\gamma) \\
c(v) & v>d(\gamma)
\end{array}\right. \\
& \text { where } \\
& \text { - }\left\{\begin{array}{ll}
d(\gamma)=d & \gamma<1 \\
d(1)=0 & \gamma=1
\end{array} \quad d: d \geq 0 \quad \text { such that } \varphi(0, d) d=M P(0, d)\right. \\
& \text { - } \quad c(v) \text { is strictly increasing on }[d(\gamma), 1]
\end{aligned}
$$

The equilibrium bid function potentially has a flat segment in the beginning. Intuitively, the local bidder might find it optimal to free-ride on the other local bidder because the probability of winning is strictly greater than zero for a local bidder with a zero bid.

Proposition 0. The equilibrium bid function of local bidders under the VCG pricing rule is given by $\beta(v)=v$.

Proof: Well known.

\section{Main Results}

This section contains our main results. In order to derive equilibrium bids explicitly, we assume uniform distributions for all values. Namely, $f($.$) is a uniform density on [0,1]$ and $g($.$) is a$ uniform density on $[0,2]$. Under this assumption, there is symmetry between global and local sides of the market because under full efficiency the global and local sides are expected to win equally often.

We start by considering the Proxy Rule.

Proposition 1. The equilibrium bid function of local bidders (in symmetric Bayesian-Nash equilibria) under the Proxy Rule is given by: 


$$
\beta(v)=\left\{\begin{array}{cc}
0 & v \leq d(\gamma) \\
1+\frac{\ln (\gamma+(1-\gamma) v)}{1-\gamma} & v>d(\gamma)
\end{array} \quad \text { if } \quad \gamma<1^{15} \quad \text { and } \quad \beta(v)=v \text { if } \quad \gamma=1\right. \text {, }
$$

where $d(\gamma)=\frac{e^{-(1-\gamma)}-\gamma}{1-\gamma}>0 \quad \forall \gamma<1$.

\section{Proof: See Appendix A.}

Figure 3 (left panel) provides examples of equilibrium bid functions for the proxy rule. In equilibrium, local bidders with low values prefer to bid zero in an attempt to free-ride. Moreover, the size of the zero-bid interval is magnified by the proxy rule itself because a local bidder with a sufficiently small bid would be required to pay her bid whenever the locals win with the other local bidder paying the rest. To put it differently, a local bidder with a small bid in the proxy auction has shading incentives which are similar to that of the first-price package auction. In sharp contrast, a high-type local bidder bids almost truthfully because she expects to be the highest bidder from the local side in which case her payment is independent from her bid.

With the increase in correlation, the zero-bid interval vanishes since a low-type local bidder no longer expects a sufficiently high bid from the other local bidder. Instead she expects a comparably low bid which makes her reluctant to shade. At the extreme case of perfect correlation, both local bidders bids truthfully in a symmetric equilibrium. The case of perfect correlation is very interesting since the proxy rule is able to achieve the first-best by combining equilibrium truthful-bidding property with the core property.

However, the proxy rule model with perfect correlation also has a multiplicity of other, asymmetric equilibria where revenue and efficiency performance is undermined. Specifically, one of the asymmetric equilibria results in truthful bidding by one of the local bidders and bidding zero by the other local bidder.

Proposition 2. The equilibrium bid function of local bidders under the Nearest-Vickrey Rule is given by:

$$
\beta(v)=\left\{\begin{array}{cc}
0 & v \leq d(\gamma) \\
k(\gamma) v-d(\gamma) & v>d(\gamma)
\end{array} \quad \text { if } \quad \gamma<1^{16} \quad \text { and } \quad \beta(v)=\frac{2}{3} v \quad \text { if } \quad \gamma=1\right.
$$

\footnotetext{
${ }^{15}$ A symmetric Bayesian-Nash equilibrium for the Proxy Rule with local bidders having independent values, i.e. $\gamma=0$, was derived independently in Sano (2010).

${ }^{16}$ A symmetric Bayesian-Nash equilibrium for Nearest-Vickrey Rule with local bidders having independent values, i.e. $\gamma=0$, was derived independently in Goeree and Lien (2009)
} 
where $\quad k(\gamma)=\frac{2}{2+\gamma} \quad d(\gamma)=\frac{2\left(\sqrt{-\gamma^{2}+2 \gamma+8}-3\right)}{\gamma^{2}+\gamma-2}>0 \quad \forall \gamma<1$.

Proof: See Appendix A.

The equilibrium bid functions for the nearest-Vickrey rule are shown in Figure 3 (central panel). The size of the zero-bid interval is smaller when comparing to the proxy rule which, as was mentioned above, induces first-price incentives to bidders with low valuations. In contrast with the proxy rule, a high-type local bidder has no incentive to bid truthfully anymore since her bid affects the price considerably. It is worth highlighting a nice linear functional form of the equilibrium bids in case of nearest-Vickrey rule where correlation parameter $\gamma$ defines the slope and intercept coefficients. For example, a local bidder shades uniformly across all values when there is no correlation between local bidders, i.e. $\gamma=0$. Positive correlation has an ambiguous effect on revenue and efficiency since it reduces bid-shading for low-type bidders and increases bid-shading for high-type bidders.

Proposition 3. The equilibrium bid function of local bidders under the Proportional Rule is given by:

$$
\begin{aligned}
& \beta(v)=\left\{\begin{array}{cc}
0 & v \leq d(\gamma) \\
k(\gamma) v-d(\gamma) & v>d(\gamma)
\end{array} \text { if } \quad \gamma<1 \quad \text { and } \quad \beta(v)=\frac{2}{3} v \quad \text { if } \quad \gamma=1\right. \text {, } \\
& \text { where } \quad k(\gamma)=\frac{2}{2+\gamma} \quad d(\gamma)=\frac{2\left(\sqrt{-\gamma^{2}+2 \gamma+8}-3\right)}{\gamma^{2}+\gamma-2}>0 \quad \forall \gamma<1 .
\end{aligned}
$$

Proof: See Appendix A.

Surprisingly, the equilibrium bid strategies for our model are the same under the nearest-Vickrey pricing rule and the proportional pricing rule. This result is mainly driven by two of our modeling assumptions: uniform distribution of the global bidder's value with the zero lower bound and the number of local bidders. In a model with more than two items for sale (discussed in greater detail in Section 5), this rule results in different equilibrium bid functions.

Proposition 4. The equilibrium bid function of local bidders under the Nearest-Bid Rule is given by:

$$
\beta(v)=\frac{1}{1-\gamma}[\ln (2)-\ln (2-(1-\gamma) v)] \quad \text { if } \quad \gamma<1 \quad \text { and } \quad \beta(v)=\frac{1}{2} v \quad \text { if } \quad \gamma=1
$$

Proof: See Appendix A.

Figure 3 (right panel) demonstrates examples of equilibrium bid functions for the nearest-bid rule. The bidding behavior under this rule is very different from the rules already considered. First, the equilibrium bid functions are strictly increasing for all correlation levels. The absence of the zero-bid interval for low-type local bidders is easily explained by the nature of the nearest-bid rule. 
Conditional on winning, the expected payment of a low-type local bidder is close to zero since the half of the refund to which the bidder is entitled almost surely exceeds the amount of her bid. At the same time, a high-type bidder shades substantially, since her payment depends heavily on the amount of her bid. Second, correlation has a strong negative impact on the equilibrium bidding functions.
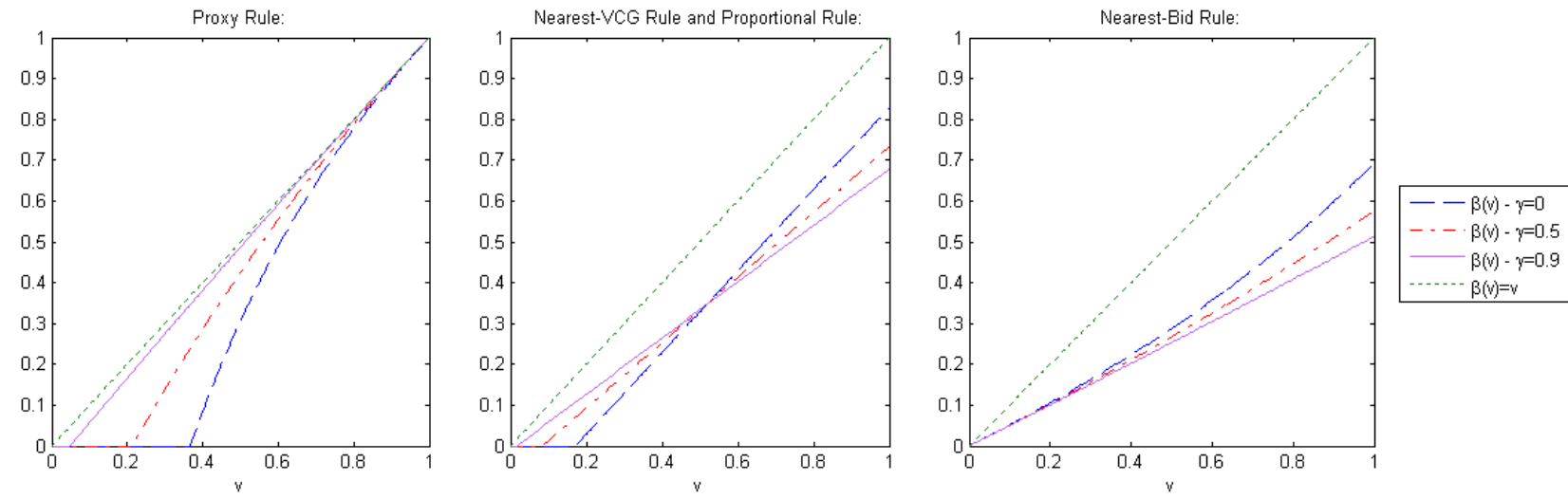

Figure 3. Equilibrium Bids: Proxy Auction Rule (left), Nearest-Vickrey (center) and Nearest-Bid (right)

Figure 2, already seen in the Introduction, summarizes the expected revenue and efficiency results for all pricing rules. The revenue of the VCG rule is negatively affected by positive correlation. By contrast, the performance of the proxy rule improves rapidly as the correlation increases, allowing the proxy rule to outperform the VCG rule in terms of revenue for a substantial range of $\gamma$ values. Moreover, the proxy rule achieves full efficiency when the locals' values are perfectly correlated. The performance of the nearest-Vickrey rule seems to be robust to correlation. This suggests that the seller interested in stable revenue and efficiency outcome across different correlation levels might have a good reason to use the nearest-Vickrey rule. However, for this particular model and distributions the proxy rule dominates other core-selecting rules including nearest-Vickrey rule. The performance of the nearest-bid rule falls with correlation, which makes the rule inferior and impractical for this environment. Corresponding numbers for revenue, efficiency and profits of bidders can be found in Table 1. Expressions used to compute revenue and efficiency for all pricing rules are provided in Appendix A. We use simulation results from Baranov (2010) in order to compare all mechanisms considered here with the first-price package auction. Interestingly, the firstprice package auction consistently beats the nearest-Vickrey and nearest-bid formats in terms of both revenue and efficiency for any positive correlation value. However, its expected revenue is lower than that of VCG and proxy auction for low and high correlation values respectively. 


\begin{tabular}{|l|l|c|c|c|c|c|}
\hline$\gamma$ & Statistics & VCG & Proxy & N-VCG & N-Bid & First-Price ${ }^{17}$ \\
\hline$\gamma=0$ & Revenue & 0.5833 & 0.5360 & 0.5327 & 0.5 & 0.5471 \\
& Efficiency & 1 & 0.8679 & 0.8431 & 0.8069 & 0.8754 \\
& Profit Global & 0.2916 & 0.4642 & 0.4673 & 0.5 & 0.4267 \\
& Profit Local & 0.2087 & 0.1342 & 0.1335 & 0.1253 & 0.1498 \\
\hline$\gamma=0.5$ & Revenue & 0.5417 & 0.5852 & 0.52 & 0.4521 & 0.5414 \\
& Efficiency & 1 & 0.9261 & 0.8356 & 0.7739 & 0.9036 \\
& Profit Global & 0.3126 & 0.4148 & 0.4798 & 0.5479 & 0.4297 \\
& Profit Local & 0.2295 & 0.1523 & 0.1415 & 0.1252 & 0.1649 \\
\hline$\gamma=1$ & Revenue & 0.5 & 0.6667 & 0.5185 & 0.4167 & 0.5411 \\
& Efficiency & 1 & 1 & 0.8334 & 0.75 & 0.9049 \\
& Profit Global & 0.3335 & 0.3335 & 0.4816 & 0.5834 & 0.4304 \\
& Profit Local & 0.2499 & 0.1666 & 0.1481 & 0.125 & 0.1757 \\
\hline
\end{tabular}

Table 1. Revenue, Efficiency and Profits

\section{Some Extensions}

\subsection{Non-Uniform Model}

The main results of this paper were derived under the assumption of uniform distributions for bidders' values. In this subsection we consider a more general model where the underlying distribution for the local bidders' values allows varying the full-efficiency frequency of winning between the global bidder and local bidders. Specifically, we assume that the cumulative distribution function for local bidders is $F(v)=v^{\alpha}, \alpha>0$ on the interval $[0,1]$. We continue to assume that the values of the local bidders are perfectly correlated with probability $\gamma$ and that the global bidder draws her value independently from the uniform distribution on $[0,2]$.

The parameter $\alpha$ of the local bidders' distribution function can be interpreted in the following way. When $\alpha$ is less then one, the sum of the local bidders' values is expected to be small in comparison with the expected value of the global bidder, implying that the local bidders lose more frequently under full efficiency. When $\alpha$ is greater then one, the situation is reversed, with the global bidder winning less frequently under truthful bidding. In other words, a high $\alpha$ makes the local bidders the stronger side in terms of their expected value.

In general, there are no closed-form solutions for this model, but it can be easily solved by appropriate numerical methods. For example, the equilibrium bidding function for the nearestVickrey rule, as in the uniform model, is linear with the slope coefficient being derived explicitly while the intercept term is determined from a non-linear equation which can be solved by a standard numerical procedure like the Newton method. The equilibrium bidding functions for the proxy rule and nearest-bid rule can be easily approximated by numerical methods for solving ODEs. Appendix $\mathrm{B}$ contains some equilibrium bidding functions and corresponding equations for numerical approximations for all pricing rules and all correlation levels.

\footnotetext{
${ }^{17}$ Simulations are based on numerical solutions developed in Baranov (2010)
} 
For the various second-price-like core-selecting auction formats, an increase in $\alpha$ leads to an increase in bid shading by the local bidders. Intuitively, a local bidder expects a higher bid from the other local bidder and tries to free-ride, reducing her bid accordingly. Symmetrically, smaller $\alpha$ results in more truthful bidding since opportunities for free-riding are reduced.

Figures 4 and 5 contain revenue and efficiency calculations for scenarios where $\alpha=2$ and $\alpha=1 / 2$, respectively. As can be seen in Appendix B, the calculations for $\alpha=2$ for the proxy auction and nearest-bid rule are based on explicit closed-form solutions of equilibria and for the nearest-VCG rule are based on "almost-closed-form" solutions. Meanwhile, the value $\alpha=1 / 2$ was chosen for symmetric comparison with $\alpha=2$; most of the associated calculations are based on numerical simulations. Even though a low value for $\alpha$ generates more sincere bids by local bidders, the expected total bid from them is smaller than in case of $\alpha=1$ (uniform distribution) and so expected seller revenue is lower. The seller revenue is affected positively by an increase in $\alpha$ for all core-selecting rules. On the other hand, an increase in $\alpha$ negatively affects revenue of the VCG auction since it leads to an increase in the probability of low revenue and zero revenue outcomes. As can be seen from Figures 4 and 5, the revenue performance of Vickrey rule relative to any core-selecting rule falls with $\alpha$. For example, the proxy rule and the nearest-Vickrey rule generate higher revenues for any correlation level $(\forall \gamma)$ when $\alpha=2$.
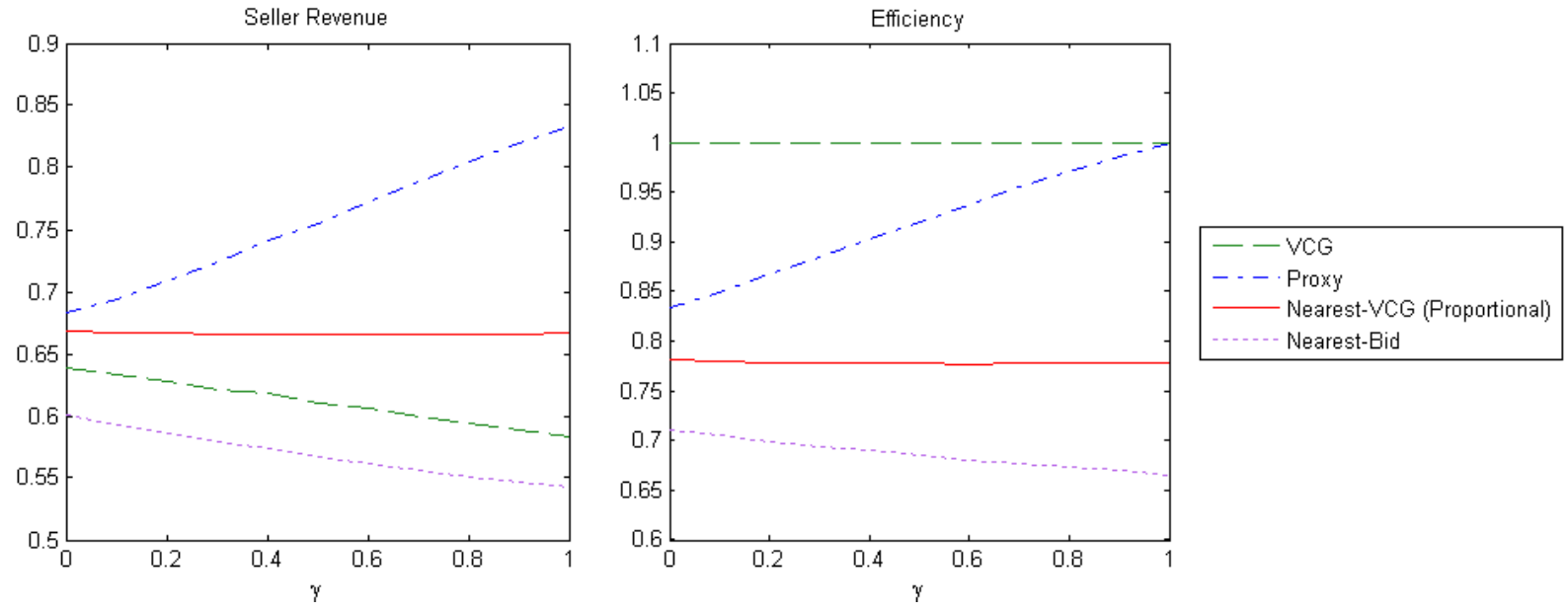

Figure 4. Seller Revenue and Efficiency for $\alpha=2$ and all $\gamma \in[0,1]$ 

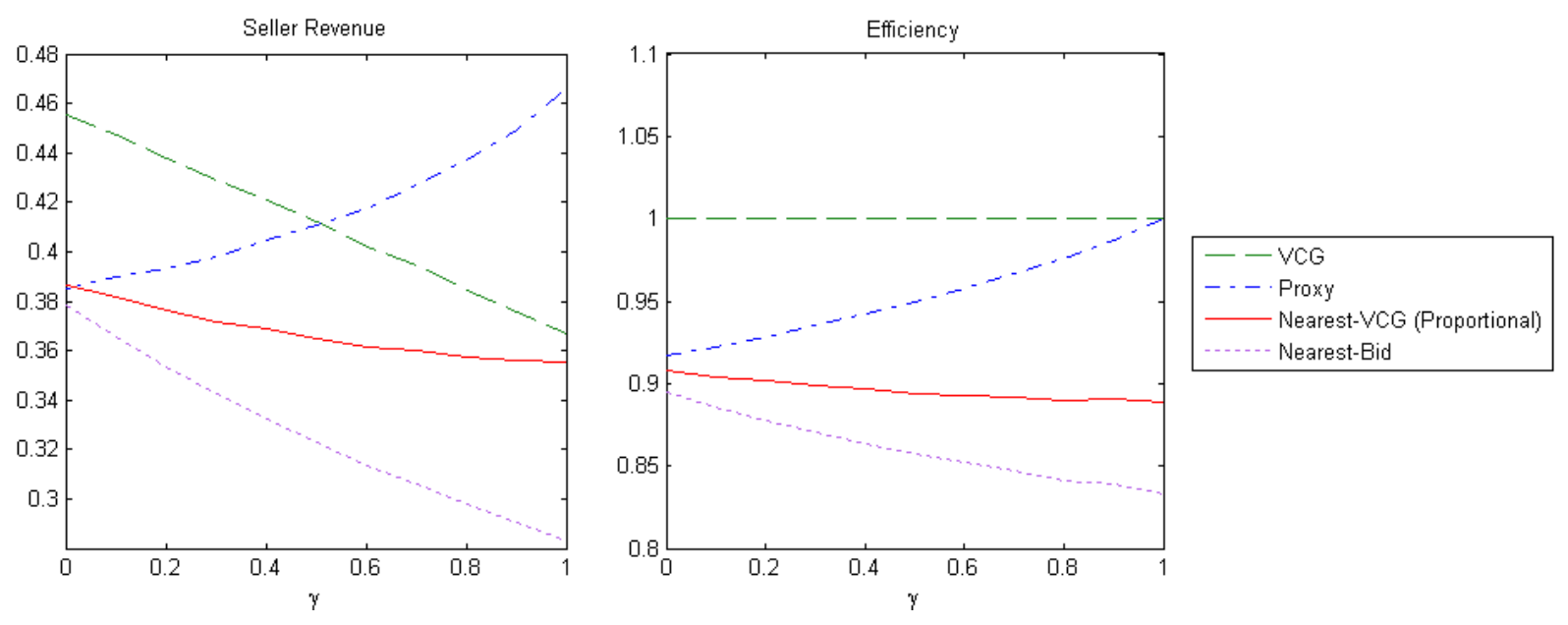

Figure 5. Seller Revenue and Efficiency for $\alpha=1 / 2$ and all $\gamma \in[0,1]$

\subsection{Number of Bidders}

Here we look into a question of robustness of our results with respect to an increase in the number of bidders. There are several interesting modifications of our model one can consider.

First of all, an increase in the number of global bidders can be modeled as a replacement of the distribution function of the global bidder with the extreme value distribution function of values. For example, if there are two global bidders who draw their values from distribution described by a function $H(u)$ independently from each other and local bidders, a version of the model with one global bidder can be used instead with distribution function of the global bidder being equal to the product of individual distributions, i.e. $G(u)=H^{2}(u)$. This replacement works because global bidders still have a weakly dominant strategy to bid truthfully. Since the distribution of this pseudo global bidder is no longer uniform, the equilibrium bidding functions of local bidders in general have to be approximated numerically.

Another interesting comparative statics exercise is to increase the number of local bidders together with the number of items offered for sale. For example, consider an auction where three items are offered, with three local bidders who only wish to acquire one item each and a global bidder who is interested only in winning all three items. Keeping a similar value structure, it is possible to solve this model for some correlation levels and some pricing rules. For example, for the proportional rule this model can be solved in closed form for all levels of correlation among local bidders' values. Unfortunately, some pricing rules such as the nearest-Vickrey rule become inherently complex in this environment. Luckily, the solution for the proportional rule sheds some light on the revenue and efficiency performance of the core-selecting rules relative to that of the Vickrey rule. An increase in the number of local bidders leads to a more severe coordination problem among them, more bid shading, and lower seller revenues and efficiency in comparison with the Vickrey rule. This finding suggests that any core-selecting rule may be a poor choice for environments where the presence of a coordination problem is significant. 
Finally, one can think of increasing the number of local bidders without increasing the number of products offered in the auction. In such environments, local bidders face competitors for their own item or market and they bid more aggressively. For example, a zero-bid interval (interval of values for which local bidder submits zero bid) no longer exists.

\subsection{Robustness Check}

In this subsection we demonstrate numerically that the partial correlation model for local bidders' values used in this paper results in equilibrium bidding functions which are qualitatively very general. Consider the following modification to the original model of Section 2. Let $M$ be a common unknown distributional factor for local bidders which is distributed on the interval $[0,1]$ with some positive density $f_{M}(m)$. Conditional on a particular realization, $m$, of the distributional factor, values for local bidders are drawn independently from a truncated logit distribution on $[0,1]$ with parameters $(m, \sigma)$, where $\sigma>0$ is a known scale factor, i.e.:

$$
f_{L}\left(v_{j} \mid M=m\right)=\frac{A(m, \sigma) e^{-\left(v_{j}-m\right) / \sigma}}{\sigma\left(1+e^{-\left(v_{j}-m\right) / \sigma}\right)^{2}} \text { for } v_{j} \in[0,1]
$$

where $A(m, \sigma)$ is a normalizing constant.

Since $m$ is not observable, both local bidders make inferences about the distribution of the other local bidder's value using their own values as signals about $m$. It is not hard to show that the conditional density takes the following form:

$$
f_{L}\left(v_{j} \mid v_{i}=s\right)=\frac{\int_{0}^{1} f_{L}\left(v_{j} \mid m\right) f_{L}(s \mid m) f_{M}(m) d m}{\int_{0}^{1} f_{L}(s \mid m) f_{M}(m) d m}
$$

Figure 6 contains approximations of conditional densities for different values of the signal and different levels of parameter $\sigma$ which controls the correlation in this model (taking the role of $\gamma$ in the main model of this paper). Levels of $\sigma$ are chosen such that the correlation between local bidders' values in the main model with gamma values $0,0.5$ and 0.9 and the model considered here are approximately equal to each other. 

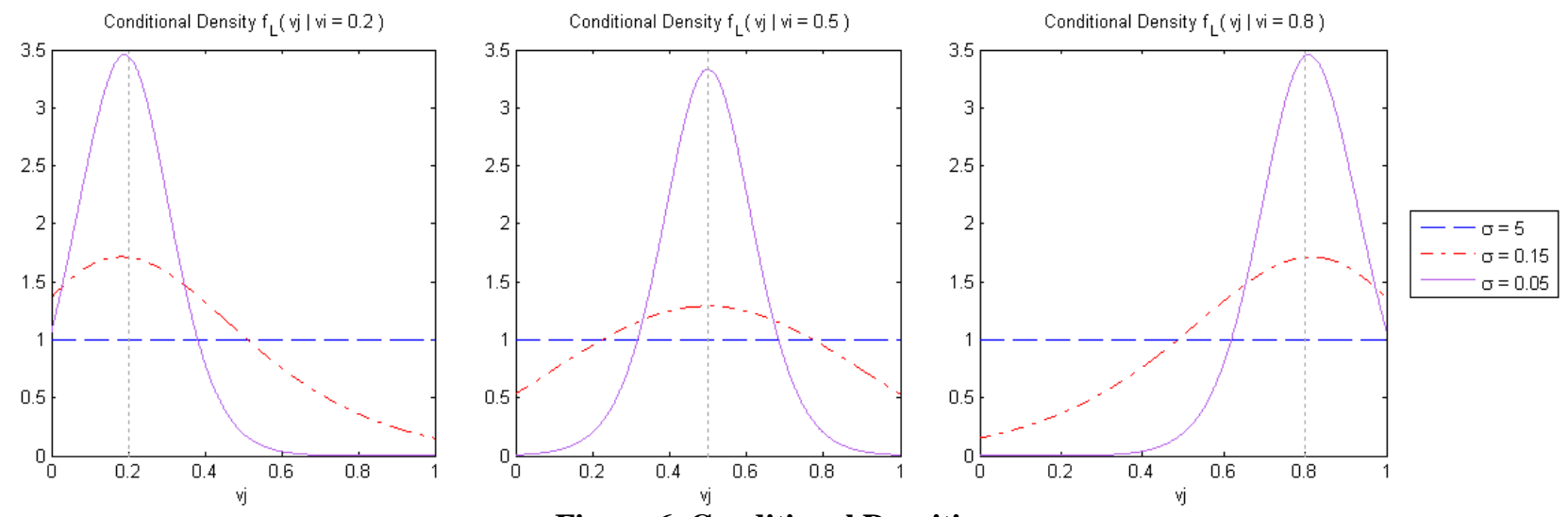

Figure 6. Conditional Densities

Given conditional densities, we approximate first-order conditions for different pricing rules derived in Lemma 3. The corresponding equilibrium bidding functions can be found at Figure 7. The numerical solutions exhibit qualitatively similar shapes and patterns as the closed-form equilibrium bidding functions derived in Section 4 (i.e. Figure 3). These results are very encouraging for the future use of this paper's partial correlation model in other contexts, since in some environments it allows us to generate closed-form solutions or extremely stable and easy numerical solutions without introducing any qualitative distinctions from smoother and more plausible partial-correlation models.
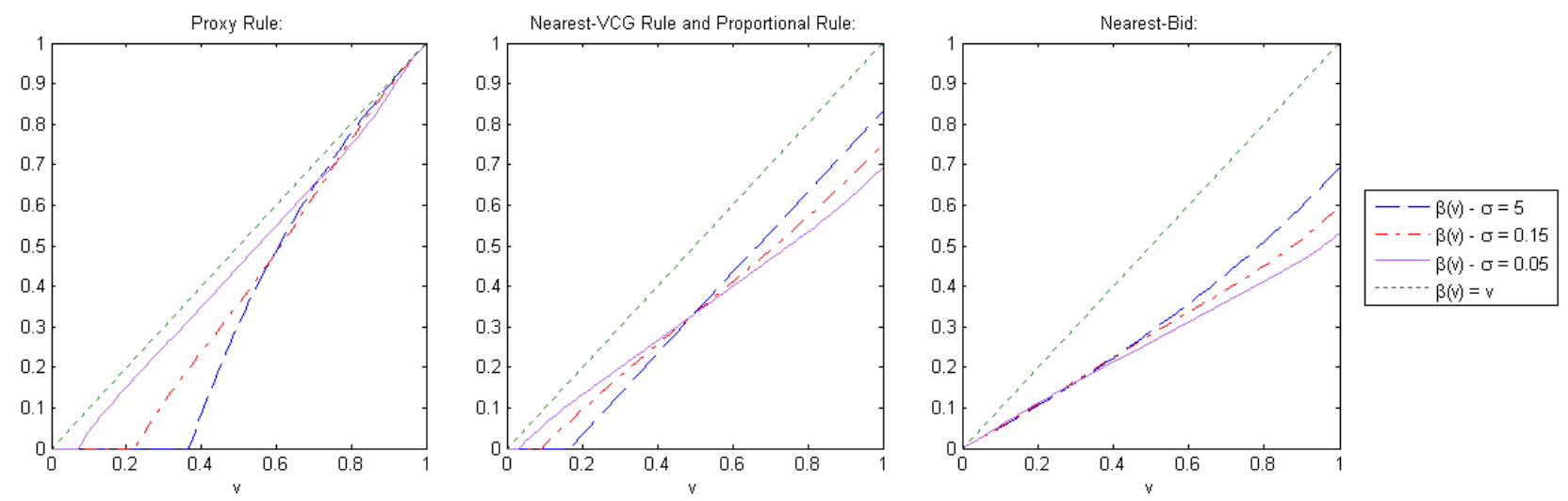

Figure 7. Approximations of Equilibrium Bids 


\section{Conclusion}

The past literature has shown the VCG mechanism to have a variety of shortcomings in environments with complementarities, including the possibility of low or even zero revenues, non-monotonicity of revenues with respect to bids and number of bidders, and vulnerability to unusual forms of collusion such as shill-bidding and collusion by losing bidders. This list of drawbacks may help to explain why this auction format - despite its attractive dominant-strategy property — is seldom used in practice: to date, we are not aware of any examples of auctions employing the Vickrey payment rule in an environment with multiple heterogeneous items. At the same time, interest in core-selecting auctions appears to be rising, with two high-stakes auctions already conducted using a two-stage version of the nearest-Vickrey pricing rule. Nevertheless, the existing literature on core-selecting auctions primarily studies complete-information environments and, to the extent that incomplete information is introduced, bidders' values are assumed to be independent.

This paper develops a model of package auctions in an environment with private information. The model considered, while simple and intuitive, includes a number of realistic features that motivate the use of package auctions, such as the presence of substantial complementarities in bidders' preferences and a positive correlation of bidders' values. We were able to derive explicit closed-form solutions for all considered payment rules and all correlation levels, under certain assumptions on distributions.

Our analysis shows that core-selecting payment rules create strong incentives for bidders without an intersection of interests to shade their bids in equilibrium. At first glance, this equilibrium property might discourage the use of core-selecting auctions, since they do not achieve full efficiency and their expected revenue might be even smaller than the revenue of the corresponding VCG auction. However, the presence of positive correlation may dramatically improve the performance of coreselecting auctions relative to the VCG mechanism. In fact, positive correlation significantly improves the performance of the proxy rule while affecting negatively the performance of the VCG. The nature of the proxy rule makes shading profitable only if the local bidder expects a sufficiently high complementary bid from the other local bidder, which becomes increasingly unlikely as the correlation increases. On the other hand, positive correlation increases the probability of low-revenue or zero-revenue outcomes in the VCG mechanism — these occur when both local bidders' are a substantial fraction of the global bidder's value.

Furthermore, the VCG mechanism has a lot of other potential drawbacks for practical applications. The full efficiency property of the VCG is actually a result of the best-case scenario analysis in a more general model of package auction where bidders can effectively use several identities (or shills) to represent their interests. If the seller has no control over identities of the bidders, the efficiency and revenue of the VCG may be significantly lower. For example, in a simple model with two items, two global bidders and a VCG pricing rule, the truth-telling strategies no longer form equilibria when one or both global bidders can enter the auction using two local shills. In contrast, for a completeinformation environment, Day and Migrom (2008) show that core-selecting auctions preserve monotonicity of revenue with respect to the number of bidders, suggesting that bidders will not be 
able to take advantage of the shill-bidding strategies when the auction utilizes a core-selecting pricing rule. In the example above, any bidder-optimal core-selecting auction has an equilibrium in sincere strategies with full efficiency, while VCG does not. Moreover, in important applications such as spectrum auctions, it is very likely that substantial correlations in bidders' valuations may be present. Thus, unlike Goeree and Lien (2009), we conclude that there may be good reasons for policymakers to select a core-selecting auction rather than a VCG mechanism.

A curious reader might notice that our paper only considers the case of positive correlation between local bidders' values without considering the case of negative correlation. This treatment seems to be satisfactory since we are not aware of any reasonable practical application for an auction model with negative correlations. The received wisdom in auction theory is that the higher the value of the object to one bidder, the higher the value of the object to any other bidder. Nevertheless, from a methodological viewpoint, a similar model of negative correlation between local bidders' values can be easily constructed. We envision that the presence of negative correlations will improve the performance of the VCG and nearest-bid pricing rules while hurting the proxy rule. This conclusion is based on the intuition developed in Section 4 on the effect of increasing the correlation, only applied in the opposite direction. 


\section{Appendix A - Proofs}

\section{Proof of Lemma 3:}

Profit function of a local bidder $i$ :

$$
\pi_{i}\left(b_{i}, v_{i}\right)=v_{i} \Phi_{i}\left(b_{i}, v_{i}\right)-P_{i}\left(b_{i}, v_{i}\right)
$$

The first-order optimality conditions are as follows:

$$
\frac{\partial \pi_{i}\left(b_{i}^{*}, v_{i}\right)}{\partial b_{i}} \leq 0 \quad b_{i}^{*} \geq 0 \quad b_{i}^{*}\left(\frac{\partial \pi_{i}\left(b_{i}^{*}, v_{i}\right)}{\partial b_{i}}\right)=0 \quad \forall v_{i} \in[0,1]
$$

In order to get the desired form of the first-order conditions, we first compute the marginal cost of winning with a bid $b_{i}$ :

$$
\begin{aligned}
\frac{\partial P_{i}\left(b_{i}, v_{i}\right)}{\partial b_{i}}= & \frac{\partial}{\partial b_{i}}\left(\gamma \int_{b_{i}+\beta\left(v_{i}\right)>u} p_{i}\left(b_{i}, \beta\left(v_{i}\right), u\right) g(u) d u+(1-\gamma) \iint_{b_{i}+\beta\left(v_{j}\right)>u} p_{i}\left(b_{i}, \beta\left(v_{j}\right), u\right) f\left(v_{j}\right) g(u) d v_{j} d u\right) \\
= & \gamma p_{i}\left(b_{i}, \beta\left(v_{i}\right), b_{i}+\beta\left(v_{i}\right)\right) g\left(b_{i}+\beta\left(v_{i}\right)\right)+(1-\gamma) \iint_{v_{j}} p_{i}\left(b_{i}, \beta\left(v_{j}\right), b_{i}+\beta\left(v_{j}\right)\right) f\left(v_{j}\right) g\left(b_{i}+\beta\left(v_{j}\right)\right) d v_{j} \\
& +\gamma \int_{b_{i}+\beta\left(v_{i}\right)>u} p_{i}^{\prime}\left(b_{i}, \beta\left(v_{i}\right), u\right) g(u) d u+(1-\gamma) \iint_{b_{i}+\beta\left(v_{j}\right)>u} p_{i}^{\prime}\left(b_{i}, \beta\left(v_{j}\right), u\right) f\left(v_{j}\right) g(u) d v_{j} d u .
\end{aligned}
$$

Note that by Lemma 2 and the pivotal pricing property we have the following:

$$
p_{i}\left(b_{i}, \beta\left(v_{i}\right), b_{i}+\beta\left(v_{i}\right)\right)=p_{i}\left(b_{i}, \beta\left(v_{j}\right), b_{i}+\beta\left(v_{j}\right)\right)=b_{i} \quad \forall v_{i}, v_{j}
$$

Plugging the last equality back to the marginal cost of winning we get a short and intuitive form for this term:

$$
\begin{aligned}
\frac{\partial P_{i}\left(b_{i}, v_{i}\right)}{\partial b_{i}} & =b_{i}\left[\gamma g\left(b_{i}+\beta\left(v_{i}\right)\right)+(1-\gamma) \int_{v_{j}} f\left(v_{j}\right) g\left(b_{i}+\beta\left(v_{j}\right)\right) d v_{j}\right]+M P_{i}\left(b_{i}, v_{i}\right) \\
& =b_{i} \varphi_{i}\left(b_{i}, v_{i}\right)+M P_{i}\left(b_{i}, v_{i}\right)
\end{aligned}
$$

Finally, the desired form of the first-order optimality conditions:

$$
\begin{gathered}
\frac{\partial \pi_{i}\left(b_{i}^{*}, v_{i}\right)}{\partial b_{i}}=v_{i} \varphi_{i}\left(b_{i}^{*}, v_{i}\right)-\frac{\partial P_{i}\left(b_{i}, v_{i}\right)}{\partial b_{i}}=\left(v_{i}-b_{i}^{*}\right) \varphi_{i}\left(b_{i}^{*}, v_{i}\right)-M P_{i}\left(b_{i}^{*}, v_{i}\right) \\
\left(v_{i}-b_{i}^{*}\right) \varphi_{i}\left(b_{i}^{*}, v_{i}\right)-M P_{i}\left(b_{i}^{*}, v_{i}\right) \leq 0 \quad b_{i}^{*} \geq 0 \\
b_{i}^{*}\left[\left(v_{i}-b_{i}^{*}\right) \varphi_{i}\left(b_{i}^{*}, v_{i}\right)-M P_{i}\left(b_{i}^{*}, v_{i}\right)\right]=0 \quad \forall v_{i} \in[0,1]
\end{gathered}
$$

\section{Proof of Proposition 1:}

The following table summarizes marginal payments for a local bidder in all possible situations: 


\begin{tabular}{|l|l|l|l|}
\hline$\beta\left(v_{i}\right), \beta\left(v_{j}\right)$ & $u \leq 2 \underline{\beta}$ & $2 \underline{\beta}<u \leq \beta\left(v_{i}\right)+\beta\left(v_{j}\right)$ & $u>\beta\left(v_{i}\right)+\beta\left(v_{j}\right)$ \\
\hline $\begin{array}{l}\text { Perfect Correlation } \\
v_{i}=v_{j}\end{array}$ & 0 & N/A & 0 \\
\hline Independence $v_{i}<v_{j}$ & 0 & 1 & 0 \\
\hline Independence $v_{i}>v_{j}$ & 0 & 0 & 0 \\
\hline
\end{tabular}

$\left(* \underline{\beta}=\beta\left(\min \left(v_{i}, v_{j}\right)\right)\right)$

The expected marginal payment for a local bidder in equilibrium is:

$$
\begin{aligned}
& \operatorname{MP}(\beta(v), v)=\frac{(1-\gamma)}{2} \int_{v}^{1}\left(\beta\left(v_{j}\right)-\beta(v)\right) d v_{j} \\
& (v-\beta(v))=(1-\gamma) \int_{v}^{1}\left(\beta\left(v_{j}\right)-\beta(v)\right) d v_{j}
\end{aligned}
$$

Note that $\beta(v)=v$ in case of $\gamma=1$. The associated expected revenue and efficiency are

$$
R^{\text {Proxy }}=2 / 3 \quad E f^{\text {Proxy }}=1
$$

For the case $\gamma<1$ the equivalent differential equation and terminal condition are given by:

$$
\beta^{\prime}=\frac{1}{\gamma+(1-\gamma) \nu} \quad \beta(1)=1
$$

The solution for this differential equation yields the equilibrium bid function.

The associated expected revenue and efficiency are given by:

$$
\begin{aligned}
& R^{\text {Proxy }}=\frac{6 e^{-(1-\gamma)}-e^{-2(1-\gamma)}-\left(1+5 \gamma-2 \gamma^{2}+\gamma^{3}\right)}{2(1-\gamma)^{3}} \quad \forall \gamma<1 \\
& E f^{\text {Proxy }}=\frac{2 e^{-(1-\gamma)}+\left(1-4 \gamma+\gamma^{2}\right)}{2(1-\gamma)^{2}}
\end{aligned}
$$

\section{Proof of Proposition 2:}

The following table summarizes marginal payments for a local bidder in all possible situations:

\begin{tabular}{|l|l|l|l|}
\hline$\beta\left(v_{i}\right), \beta\left(v_{j}\right)$ & $u \leq \beta\left(v_{i}\right)$ & $\beta\left(v_{i}\right)<u \leq \beta\left(v_{i}\right)+\beta\left(v_{j}\right)$ & $u>\beta\left(v_{i}\right)+\beta\left(v_{j}\right)$ \\
\hline $\begin{array}{l}\text { Perfect Correlation } \\
v_{i}=v_{j}\end{array}$ & 0 & $1 / 2$ & 0 \\
\hline Independence & 0 & $1 / 2$ & 0 \\
\hline
\end{tabular}


The expected marginal payment for a local bidder in equilibrium is:

$$
\begin{aligned}
& M P(\beta(v), v)=\frac{1}{4}\left[\gamma \beta(v)+(1-\gamma) \int_{0}^{1} \beta\left(v_{j}\right) d v_{j}\right] \\
& (v-\beta(v))=\frac{1}{2}\left[\gamma \beta(v)+(1-\gamma) \int_{0}^{1} \beta\left(v_{j}\right) d v_{j}\right]
\end{aligned}
$$

Note that $\beta(v)=2 / 3 v$ in case of $\gamma=1$. The associated expected revenue and efficiency are:

$$
R^{\text {Nearest-VCG }}=14 / 27 \quad E f^{\text {Nearest-VCG }}=5 / 6
$$

If $\gamma<1$ :

$$
\beta(v)=\frac{2}{2+\gamma}\left[v-\frac{(1-\gamma)}{2} \int_{0}^{1} \beta\left(v_{j}\right) d v_{j}\right]=k(\gamma) v-d(\gamma)
$$

The associated expected revenue and efficiency are given by:

$$
\begin{gathered}
R^{\text {Nearest-VCG }}=\frac{(d-1)\left(-3 d^{3}(1-\gamma)(1+\gamma)^{2}+13 d^{2}(1+\gamma)+d\left(7+25 \gamma+12 \gamma^{2}\right)-11 \gamma-17\right)}{6(2+\gamma)^{2}} \\
E f^{\text {Nearest-VCG }}=\frac{2 d^{2}(1+\gamma)-2 d(2+\gamma)+\gamma+4}{2(2+\gamma)}, \\
\text { where } d(\gamma)=\frac{2\left(\sqrt{-\gamma^{2}+2 \gamma+8}-3\right)}{\gamma^{2}+\gamma-2} \text { and } \gamma<1 .
\end{gathered}
$$

\section{Proof of Proposition 3:}

The following table summarizes marginal payments for a local bidder in all possible situations:

\begin{tabular}{|l|l|l|}
\hline$\beta\left(v_{i}\right), \beta\left(v_{j}\right)$ & $0<u \leq \beta\left(v_{i}\right)+\beta\left(v_{j}\right)$ & $u>\beta\left(v_{i}\right)+\beta\left(v_{j}\right)$ \\
\hline $\begin{array}{l}\text { Perfect Correlation } \\
v_{i}=v_{j}\end{array}$ & $\frac{\beta\left(v_{i}\right) u}{4 \beta\left(v_{i}\right)^{2}}$ & 0 \\
\hline Independence & $\frac{\beta\left(v_{j}\right) u}{\left(\beta\left(v_{i}\right)+\beta\left(v_{j}\right)\right)^{2}}$ & 0 \\
\hline
\end{tabular}

The expected marginal payment for a local bidder in equilibrium is: 


$$
M P(\beta(v), v)=\frac{1}{4}\left[\gamma \beta(v) \frac{(2 \beta(v))^{2}}{4 \beta(v)^{2}}+(1-\gamma) \int_{0}^{1} \beta\left(v_{j}\right) \frac{\left(\beta(v)+\beta\left(v_{j}\right)\right)^{2}}{\left(\beta(v)+\beta\left(v_{j}\right)\right)^{2}} d v_{j}\right]=\frac{1}{4}\left[\gamma \beta(v)+(1-\gamma) \int_{0}^{1} \beta\left(v_{j}\right) d v_{j}\right]
$$

Note that the expected marginal payment is exactly the same as the one for Nearest-VCG Payment

Rule. Therefore, equilibrium bid function, expected revenue and efficiency are the same.

Proof of Proposition 4:

The following table summarizes marginal payments for a local bidder in all possible situations:

\begin{tabular}{|l|l|l|l|}
\hline$\beta\left(v_{i}\right), \beta\left(v_{j}\right)$ & $u \leq \bar{\beta}-\underline{\beta}$ & $\bar{\beta}-\underline{\beta}<u \leq \beta\left(v_{i}\right)+\beta\left(v_{j}\right)$ & $u>\beta\left(v_{i}\right)+\beta\left(v_{j}\right)$ \\
\hline $\begin{array}{l}\text { Perfect Correlation } \\
v_{i}=v_{j}\end{array}$ & N/A & $1 / 2$ & 0 \\
\hline Independence & 0 & $1 / 2$ & 0 \\
\hline
\end{tabular}

$\left(* \beta=\beta\left(\min \left(v_{i}, v_{j}\right)\right) \quad \bar{\beta}=\beta\left(\max \left(v_{i}, v_{j}\right)\right)\right)$

The expected marginal payment for a local bidder in equilibrium is:

$$
\begin{aligned}
& M P(\beta(v), v)=\frac{\gamma}{2} \beta(v)+\frac{(1-\gamma)}{2}\left[\int_{0}^{v} \beta\left(v_{j}\right) d v_{j}+\int_{v}^{1} \beta(v) d v_{j}\right]=\frac{\gamma}{2} \beta(v)+\frac{(1-\gamma)}{2}\left[\int_{0}^{v} \beta\left(v_{j}\right) d v_{j}+\beta(v)(1-v)\right] \\
& (v-\beta(v))=\gamma \beta(v)+(1-\gamma)\left[\int_{0}^{v} \beta\left(v_{j}\right) d v_{j}+\beta(v)(1-v)\right]
\end{aligned}
$$

Note that $\beta(v)=\frac{1}{2} v$ in case of $\gamma=1$. The associated expected revenue and efficiency are:

$$
R^{\text {Nearest-Bid }}=5 / 12 \quad E f^{\text {Nearest-Bid }}=3 / 4
$$

For the case $\gamma<1$ the equivalent differential equation and initial condition are given by:

$$
\beta^{\prime}=\frac{1}{2-(1-\gamma) \nu} \quad \beta(0)=0
$$

The associated expected revenue and efficiency are given by: 


$$
\begin{aligned}
& R^{\text {Nearest-Bid }}=\frac{1+\gamma(\gamma(5+4 \ln 2)-6+4 \ln 2)-4 \gamma(1+\gamma) \ln (1+\gamma)}{2(1-\gamma)^{3}} \\
& E f^{\text {Nearest-Bid }}=\frac{3-4 \gamma+\gamma^{2}+2(1+\gamma)(\ln (1+\gamma)-\ln 2)}{2(1-\gamma)^{2}}
\end{aligned}
$$




\section{Appendix B - Solution Summary}

1) Proxy Rule

\begin{tabular}{|c|c|c|}
\hline $\begin{array}{l}\text { Local Distribution Parameter } \\
\alpha>0\left(F(v)=v^{\alpha}, \alpha>0\right)\end{array}$ & $\begin{array}{l}\text { Correlation Parameter } \\
0 \leq \gamma \leq 1\end{array}$ & Symmetric Equilibrium Bid \\
\hline$\alpha>0$ & $\gamma=1$ & $b(v)=v$ \\
\hline$\alpha=1$ & $0 \leq \gamma<1$ & $b(v)=\max \left(0,1+\frac{\ln (\gamma+(1-\gamma) v)}{1-\gamma}\right)$ \\
\hline$\alpha \neq 1$ & $\gamma=0$ & $b(v)=\max \left(0, \frac{v^{1-\alpha}-\alpha}{1-\alpha}\right)$ \\
\hline$\alpha=2$ & $0<\gamma<1$ & $\begin{array}{l}b(v)=\max \left(0, \frac{1}{\sqrt{\gamma(1-\gamma)}} \tan ^{-1}\left(\sqrt{\frac{1-\gamma}{\gamma}} v\right)+C\right) \\
C=1-\frac{1}{\sqrt{\gamma(1-\gamma)}} \tan ^{-1}\left(\sqrt{\frac{1-\gamma}{\gamma}}\right)\end{array}$ \\
\hline$\alpha \neq 1, \alpha \neq 2$ & $0<\gamma<1$ & $\begin{array}{l}\text { No closed-form solution. } \\
\text { Differential Equation: } \\
b^{\prime}=\frac{1}{\gamma+(1-\gamma) v^{\alpha}} \quad b(1)=1\end{array}$ \\
\hline
\end{tabular}

2) Nearest-VCG Rule (Proportional Rule)

\begin{tabular}{|c|c|c|}
\hline $\begin{array}{l}\text { Local Distribution Parameter } \\
\alpha>0\left(F(v)=v^{\alpha}, \alpha>0\right)\end{array}$ & $\begin{array}{l}\text { Correlation Parameter } \\
0 \leq \gamma \leq 1\end{array}$ & Symmetric Equilibrium Bid \\
\hline$\alpha>0$ & $\gamma=1$ & $b(v)=2 / 3 v$ \\
\hline$\alpha=1$ & $0 \leq \gamma<1$ & $\begin{array}{l}\beta(v)=\left\{\begin{array}{cc}k v-d & v>\frac{d}{k} \\
0 & v \leq \frac{d}{k}\end{array}\right. \\
k=\frac{2}{2+\gamma} \quad d=\frac{3 k^{2}-2 k \sqrt{3 k-1}}{3 k-2}\end{array}$ \\
\hline$\alpha \neq 1$ & $\gamma=0$ & $\begin{array}{l}\text { Almost-Closed Form: } \\
\beta(v)=\left\{\begin{array}{cc}k v-d & v>\frac{d}{k} \\
0 & v \leq \frac{d}{k}\end{array}\right.\end{array}$ \\
\hline
\end{tabular}




\begin{tabular}{|l|l|l|}
\hline$\alpha \neq 1$ & $0<\gamma<1$ & $\begin{array}{l}k=\frac{2}{2+\gamma} \\
\mathrm{d} \text { is defined by equation: } \\
\frac{d^{\alpha+1}}{(1+\alpha) k^{\alpha}}-\frac{3 k}{3 k-2} d+\frac{\alpha k}{\alpha+1}=0 \\
\text { Note that the equation has exactly one } \\
\text { root on }(0, \mathrm{k}) \text { interval }\end{array}$ \\
\hline
\end{tabular}

3) Nearest-Bid Rule

\begin{tabular}{|c|c|l|}
\hline $\begin{array}{l}\text { Local Distribution Parameter } \\
(F>0\end{array}$ & $\left.\left.\begin{array}{l}\text { Correlation Parameter } \\
0 \leq \gamma \leq 1\end{array}\right) v^{\alpha}, \alpha>0\right)$ & Symmetric Equilibrium Bid \\
\hline$\alpha>0$ & $\gamma=1$ & $b(v)=1 / 2 v$ \\
\hline$\alpha=1$ & $0 \leq \gamma<1$ & $b(v)=\frac{1}{1-\gamma}[\ln (2)-\ln (2-(1-\gamma) v)]$ \\
\hline$\alpha=2$ & $0 \leq \gamma<1$ & $b(v)=\frac{1}{\sqrt{2(1-\gamma)}} \ln \mid \frac{(1-\gamma) v+\sqrt{2(1-\gamma)} \mid}{(1-\gamma) v-\sqrt{2(1-\gamma)} \mid}$ \\
\hline$\alpha \neq 1, \alpha \neq 2$ & $\gamma=0$ & $\begin{array}{l}\text { No closed-form solution. } \\
\text { Differential Equation: } \\
b^{\prime}=\frac{1}{2-(1-\gamma) v^{\alpha}} \quad b(0)=0\end{array}$ \\
\hline$\alpha \neq 1, \alpha \neq 2$ & $0<\gamma<1$ & \multicolumn{2}{|c}{} \\
\hline
\end{tabular}




\section{References}

Ausubel, Lawrence M. (2006), “An Efficient Dynamic Auction for Heterogeneous Commodities,” American Economic Review, Vol. 96, No. 3, pp. 602-629.

Ausubel, Lawrence M., Peter Cramton, R. Preston McAfee, and John McMillan (1997), "Synergies in Wireless Telephony: Evidence from the Broadband PCS Auctions," Journal of Economics and Management Strategy, 6:3, 497-527.

Ausubel, Lawrence M., Peter Cramton and Paul Milgrom (2006), “The Clock-Proxy Auction: A Practical Combinatorial Auction Design," in Peter Cramton, Yoav Shoham, and Richard Steinberg (eds.), Combinatorial Auctions, Chapter 5, 115-138, MIT Press.

Ausubel, Lawrence M. and Paul R. Milgrom (2002), “Ascending Auctions with Package Bidding,” Frontiers of Theoretical Economics, 1(1), Article 1.

Ausubel, Lawrence M. and Paul R. Milgrom (2006), “Ascending Proxy Auctions”, Chapter 3 in P. Cramton, Y. Shoham, and R. Steinberg (eds.), Combinatorial Auctions, 79-98, MIT Press, 2006.

Baranov, Oleg (2010), “First-Price Package Auctions with Incomplete Information,” Working Paper, University of Maryland.

Bernheim, B. Douglas and Michael Whinston (1986), "Menu Auctions, Resource Allocation and Economic Influence,” Quarterly Journal of Economics, 101: 1-31.

Chernomaz, Kirill and Dan Levin (2008), "Efficiency and Synergy in a Multi-Unit Auction with and without Package Bidding: an Experimental Study”, Working Paper, Ohio State University.

Clarke, Edward H (1971). “Multipart Pricing of Public Goods.” Public Choice, 11, pp. 17-33.

Cramton, Peter (2009), “Spectrum Auction Design”, Working Paper, University of Maryland.

Crémer, Jacques and Richard P. McLean (1985), “Optimal Selling Strategies under Uncertainty for a Discriminating Monopolist when Demands are Interdependent.” Econometrica, 53(2): 345-361.

Day, Robert W. and Cramton, Peter (2008), “The Quadratic Core-Selecting Payment Rule for Combinatorial Auctions,” Working Paper, University of Maryland.

Day, Robert W. and Milgrom, Paul R (2008), “Core-selecting Package Auctions,” International Journal of Game Theory, 36, 393-407.

Day, Robert W. and S. Raghavan (2007), "Fair Payments for Efficient Allocations in Public Sector Combinatorial Auctions," Management Science, 53, 1389-1406.

Erdil, Aytek and Klemperer, Paul (2010), “A New Payment Rule for Core-Selecting Package Auctions,” Journal of the European Economic Association (forthcoming) 
Federal Aviation Administration (2008), “2009 New York Slot Auctions Bidder Seminar,” http://www.faa.gov/about/office_org/headquarters_offices/aep/ny_auctions/media/bidder-seminar2009-new-york-slot-auctions-2.pdf.

Fox, Jeremy T. and Patrick Bajari (2009), "Measuring the Efficiency of an FCC Spectrum Auction,” Working Paper, University of Chicago.

Goeree, Jacob K. and Yuanchuan Lien (2009), “On the Impossibility of Core-Selecting Auctions,” Working Paper, Caltech.

Groves, Theodore (1973), “Incentives in Teams,” Econometrica,,41(4), pp. 617-31.

Hoffman, Karla, Dinesh Menon, Susara van den Heever and Tomas Wilson (2006), "Observations and Near-Direct Implementations of the Ascending Proxy Auction,” in Peter Cramton, Yoav Shoham, and Richard Steinberg (eds.), Combinatorial Auctions, Chapter 17, 415-450, MIT Press

Krishna, Vijay and Robert (1996), "Simultaneous Auctions with Synergies,” Games and Economic Behavior, 17, No. 1, pp. 1-31.

Parkes, David C. (2001), Iterative Combinatorial Auctions: Achieving Economic and Computational Efficiency, Ph.D. thesis, Department of Computer and Information Science, University of Pennsylvania. http://www.cis.upenn.edu/ dparkes/diss.html.

Parkes, David C. and Ungar, Lyle H. (2000) "Interative Combinational Auctions: Theory and Practice," in American Association of Artificial Intelligence, ed., Proceedings of the 17th National Conference on Artificial Intelligence (AAAI 2000). Menlo Park: AAI Press, pp. 74-81.

Sano, R. (2010), “An Equilibrium Analysis of a Package Auction with Single-Minded Bidders,” Working Paper, Graduate School of Economics, University of Tokyo.

Vickrey, William (1961), “Counterspeculation, Auctions, and Competitive Sealed Tenders.” Journal of Finance, 16(1), pp. 8-37. 